\title{
NEW SUFFICIENT CONDITIONS FOR A CENTER AND GLOBAL PHASE PORTRAITS FOR POLYNOMIAL SYSTEMS
}

\author{
H. Giacomini And M. NdiAye
}

\begin{abstract}
In this paper we consider cubic polynomial systems of the form: $\dot{x}=y+P(x, y), \dot{y}=-x+Q(x, y)$, where $P$ and $Q$ are polynomials of degree 3 without linear part. If $M(x, y)$ is an integrating factor of the system, we propose its reciprocal $V(x, y)=\frac{1}{M(x, y)}$ as a linear function of certain coefficients of the system. We find in this way several new sets of sufficient conditions for a center. The resulting integrating factors are of Darboux type and the first integrals are in the Liouville form.

By induction, we have generalized these results for polynomials systems of arbitrary degree.

Moreover, for the cubic case, we have constructed all the phase portraits for each new family with a center.
\end{abstract}

\section{Introduction}

In a previous paper $[\mathbf{1}]$, we considered the following system of differential equations $(S)$

$$
\begin{aligned}
& \dot{x}=P(x, y), \\
& \dot{y}=Q(x, y),
\end{aligned}
$$

where $P(x, y)$ and $Q(x, y)$ are polynomials of degree $n$ of the form

$$
\begin{aligned}
& P(x, y)=y+\sum_{j=2}^{n} \sum_{i=0}^{j} a_{i, j-i} x^{i} y^{j-i} \\
& Q(x, y)=-x-\sum_{j=2}^{n} \sum_{i=0}^{j} b_{i, j-i} x^{i} y^{j-i} .
\end{aligned}
$$


For this system, we found sufficient conditions for the existence of a center at the origin. This has been accomplished by proposing a constant of motion linear in some coefficients of the system.

We recall that the problem of the center plays an important role in the second part of Hilbert's $16^{\text {th }}$ problem, which asks for the maximum number of limit cycles that a vector field given by polynomials of degree $n$ should have. There are well-known necessary and sufficient conditions for quadratic systems $[\mathbf{2}],[\mathbf{3}],[\mathbf{4}]$ and for systems in which $P(x, y)$ and $Q(x, y)$ are cubic polynomials without quadratic terms [5]. Sufficient conditions are also well-known for other classes of cubic systems [6]. In [7], the author classified the reversible cubic systems with center and recently, Sokulsky began the classification of Darboux integrals for cubic systems [8].

In this paper we continue the study of the problem of the center. In section 1 we consider the general cubic system $(n=3)$. Instead of looking for a constant of motion, we work with the reciprocal of an integrating factor of $(S): V(x, y)$.

In [9] it has been shown that $V(x, y)$ has very interesting properties and that it is relevant in the analysis of the problem of limit cycles.

By studying quadratic systems, we found that, while the known constants of motion are complicated functions, the reciprocal of the integrating factor $V(x, y)$ turns out to be a simple polynomial. This has also been showed in [10]. We hoped to find the same result for the general cubic system, but unfortunately, this is not always the case. A counter-example has been given in $[\mathbf{1 1}]$, where $V(x, y)$ is not a polynomial function.

Nevertheless, we found particular cases of the general cubic system, for which the integrating factor $\frac{1}{V(x, y)}$ is of Darboux type (see [12], [13], [14]) and leads to an elementary first integral of the Liouville form [15] as we can see in the expression (34) of section $3 . V(x, y)$ is given by a fourth-degree polynomial that factorizes as a product of a polynomial of degree one and another of degree three. These two polynomials are directly related to particular algebraic integrals of the system [16].

The corresponding constant of motion associated to the integrating factor $\frac{1}{V(x, y)}$ is analytic in the neighbourhood of the origin and therefore the origin is a center.

These results have been found by proposing a function $V(x, y)$ which is linear in the coefficient $a_{21}$ of the general cubic system. In fact, our first choice was the coefficient $a_{11}$, because for quadratic systems, $V(x, y)$ is linear in this coefficient, but the calculations rapidely became very involved. After that, we have proposed a first integral linear in the 
coefficient $a_{21}$. This choice is natural and leads to new conditions for a center.

By using the fact that $V(x, y)$ satisfies the equation:

(3) $\frac{\partial V(x, y)}{\partial x} P(x, y)+\frac{\partial V(x, y)}{\partial y} Q(x, y)$

$$
-V(x, y)\left(\frac{\partial P(x, y)}{\partial x}+\frac{\partial Q(x, y)}{\partial y}\right)=0
$$

we found in this way new sufficient conditions for which the origin is a center of the general cubic system.

In section 2 , we generalize these results to polynomial systems $(S)$ of arbitrary degree $n$. This is done by proposing a function $V(x, y)$ of the form:

$$
V(x, y)=V_{1}(x, y) a_{n-1,1}+V_{2}(x, y)
$$

where $V_{1}(x, y)$ and $V_{2}(x, y)$ don't depend on $a_{n-1,1}$.

We found that, similarly to the case $n=3, V(x, y)$ factorizes as a polynomial of degree 1 and another of degree $n$, associated to two particular algebraic integrals of the system.

Finally, in section 3, we use the particular algebraic integrals for constructing global phase portraits of the system $\left(S_{1}\right)$ with $n=3$.

\section{Sufficient conditions for a center in the case $n=3$}

Here, we consider the general cubic system $\left(S_{1}\right)$

$$
\begin{aligned}
& \dot{x}=P(x, y), \\
& \dot{y}=Q(x, y),
\end{aligned}
$$

where

$$
\begin{aligned}
& P(x, y)=y+\sum_{j=2}^{3} \sum_{i=0}^{j} a_{i, j-i} x^{i} y^{j-i} \\
& Q(x, y)=-x-\sum_{j=2}^{3} \sum_{i=0}^{j} b_{i, j-i} x^{i} y^{j-i} .
\end{aligned}
$$

In order to obtain sufficient conditions for the existence of a center, we propose the reciprocal of an integrating factor $V(x, y)$ which is linear in the coefficient $a_{21}$ of $\left(S_{1}\right)$ :

$$
V(x, y)=V_{1}(x, y) a_{21}+V_{2}(x, y)
$$


where $V_{1}(x, y)$ and $V_{2}(x, y)$ are independent of $a_{21}$.

By replacing (7) in equation (3) and imposing the condition that (3) must be identically zero for arbitrary values of $a_{21}$, we obtain three equations

$$
\begin{aligned}
& u_{1}(x, y)=0, \\
& u_{2}(x, y)=0, \\
& u_{3}(x, y)=0,
\end{aligned}
$$

where

$$
u_{1}(x, y)=\frac{\partial V_{1}(x, y)}{\partial x} x^{5} y-2 V_{1}(x, y) x^{4} y,
$$

and $u_{2}(x, y)$ and $u_{3}(x, y)$ are complicated expressions in terms of $V_{1}(x, y)$, $V_{2}(x, y)$ and their partial derivatives.

From (11), we have

$$
V_{1}(x, y)=h(y) x^{2} .
$$

When we replace (12) in (9), we obtain

$$
W_{1}(x, y)+\frac{\partial V_{2}(x, y)}{\partial x} x^{5} y-2 V_{2}(x, y) x^{4} y=0,
$$

where $W_{1}(x, y)$ is a function of $x, y$ and $h(y)$.

From (13), we can calculate $V_{2}(x, y)$

$$
V_{2}(x, y)=\left(W_{2}(x, y)+h_{1}(y)\right) x^{2},
$$

where $h_{1}(y)$ is an arbitrary function of $y$ and $W_{2}(x, y)$ is a function of $x$, $y$ and $h(y)$.

If we replace (12) and (14) in (10), we obtain an equation of the form

$$
W_{3}(x, y)=0,
$$

where $W_{3}(x, y)$ is function of $x, y, h(y), h_{1}(y), h^{\prime}(y), h^{\prime \prime}(y)$ and $h_{1}^{\prime}(y)$.

With respect to the variable $x, W_{3}(x, y)$ is a polynomial in $x$ and $\log |x|$ (of first degree in $\log |x|$ ).

As (15) must be identically zero for arbitrary values of $x$, the coefficients of $\log |x|$ and $(\log |x|)^{0}$ in (15) must be identically zero. From this, we obtain two equations

$$
\begin{aligned}
& W_{4}(x, y)=0, \\
& W_{5}(x, y)=0,
\end{aligned}
$$


where $W_{4}(x, y)$ and $W_{5}(x, y)$ are polynomials in the variable $x$ of degree 7 and 5 respectively. The coefficients of these polynomials are functions of $y$ and must be identically zero. One of the coefficients of $W_{4}(x, y)$ gives the equation

(18) $\left(1+a_{20} y+a_{03} y^{2}\right)\left(-\left(b_{11}+2 b_{12}\right) h(y)+\left(1+b_{11} y+b_{12} y^{2}\right) h^{\prime}(y)\right)=0$.

As the first factor cannot be zero, the second one must vanish. This condition enable us to determine

$$
h(y)=k\left(1+b_{11} y+b_{12} y^{2}\right)
$$

where $k$ is an arbitrary constant factor.

By replacing (19) in the others coefficients of (16), we find that they are identically zero.

By replacing (19) in (17), we obtain 6 equations:

$$
\begin{gathered}
r_{1}(y)=0, \\
r_{2}(y)=0, \\
\vdots \\
r_{6}(y)=0 .
\end{gathered}
$$

Each of these equations must be identically zero for arbitrary values of $y$. One of them is a polynomial of fourth degree in $y$. As each coefficient of this polynomial must vanish, we obtain the following system of five algebraic equations between the coefficients of $\left(S_{1}\right)$ :

$$
\begin{aligned}
& b_{02}=0, \\
& 3 a_{02} b_{02}+6 b_{03}+2 b_{02} b_{11}=0, \\
& 2 a_{03} b_{02}+5 a_{02} b_{03}+a_{02} b_{02} b_{11}+4 b_{03} b_{11}=0, \\
& 4 a_{03} b_{03}+3 a_{02} b_{03} b_{11}-a_{02} b_{02} b_{11}+2 b_{03} b_{12}=0, \\
& 2 a_{03} b_{03} b_{11}-2 a_{03} b_{02} b_{12}+a_{02} b_{03} b_{12}=0 .
\end{aligned}
$$

It is easy to verify that the solution of this system is $b_{02}=b_{03}=0$, the remainder coefficients being arbitrary.

By replacing these results in the other equations of the system (20), we obtain the condition

$$
b_{11} b_{30}^{2}=0
$$

If we take $b_{11}=0$, by following the calculation, we find that $b_{30}=0$. Therefore, we must choose the solution $b_{30}=0$ and $b_{11}$ arbitrary. 
Using this result, we obtain from equations (20), after a long calculation

$$
\begin{array}{ll}
a_{02}=-2 a_{20}, & b_{02}=b_{03}=b_{30}=0, \\
a_{03}=a_{20}^{2}, & b_{11}=-a_{20}+\frac{b_{21}}{b_{20}}, \\
a_{30}=a_{20} b_{20}, & b_{12}=-\frac{a_{20} b_{21}}{b_{20}}, \\
a_{12}=-a_{20} a_{11}, & h_{1}(y)=b_{12}\left(1+b_{11} y+b_{12} y^{2}\right),
\end{array}
$$

where we supposed that $b_{20} \neq 0$.

The final expression that we obtain for $V(x, y)$ is polynomial in $x$ and $y:$

$$
\begin{aligned}
V(x, y)= & \left(b_{20}+b_{21} y\right)\left(-a_{20} b_{20}\left(a_{20} b_{20}+b_{21}\right) x^{3}\right. \\
& +\left(a_{21} b_{20}-a_{20} b_{21}\right)\left(1-a_{20} y\right) x^{2} \\
& \left.+a_{11} b_{20}\left(1-a_{20} y\right)^{2} x+b_{20}\left(1-a_{20} y\right)^{3}\right) / b_{20}^{2}
\end{aligned}
$$

We wrote $V(x, y)$ in this form in order to be able to generalize this expression for a system $(S)$ of arbitrary degree. Since $V(x, y)$ is the reciprocal of an integrating factor, we can evaluate the associated constant of motion $I(x, y)$ from the relations:

$$
\frac{\partial I(x, y)}{\partial x}=-\frac{Q(x, y)}{V(x, y)}, \quad \frac{\partial I(x, y)}{\partial y}=\frac{P(x, y)}{V(x, y)}
$$

It can be easily verified that the behaviour of $I(x, y)$ in a neighbourhood of the origin is equivalent to $\left(x^{2}+y^{2}\right) / 2$. This proves that $I(x, y)$ is analytic at the origin and the level curves in a neighbourhood of this point are circles surrounding the origin $O$. The explicit form of $I(x, y)$ is given in the Proposition 2 of section 3.

We conclude now with the following theorem:

Theorem 1.1. Consider system $\left(S_{1}\right)$ with $b_{20} \neq 0$. Under conditions

$$
\begin{array}{ll}
a_{02}=-2 a_{20}, & b_{02}=b_{03}=b_{30}=0, \\
a_{03}=a_{20}^{2}, & b_{11}=-a_{20}+\frac{b_{21}}{b_{20}}, \\
a_{30}=a_{20} b_{20}, & b_{12}=-a_{20} \frac{b_{21}}{b_{20}}, \\
a_{12}=-a_{20} a_{11}, &
\end{array}
$$


the origin is a center and the associated reciprocal of an integrating factor is a polynomial form given by

$$
\begin{aligned}
V(x, y)= & \left(b_{20}+b_{21} y\right)\left(-a_{20} b_{20}\left(a_{20} b_{20}+b_{21}\right) x^{3}\right. \\
& +\left(a_{21} b_{20}-a_{20} b_{21}\right)\left(1-a_{20} y\right) x^{2} \\
& \left.+a_{11} b_{20}\left(1-a_{20} y\right)^{2} x+b_{20}\left(1-a_{20} y\right)^{3}\right) / b_{20}^{2} .
\end{aligned}
$$

1.1. Particular case $b_{20}=0$.

By using the same procedure that above, we find the following conditions for a center:

$$
\begin{array}{ll}
a_{02}=-2 a_{20} & b_{30}=b_{02}=b_{03}=b_{21}=a_{30}=0, \\
a_{03}=a_{20}^{2} & b_{12}=-\left(a_{20}^{2}+a_{20} b_{11}\right), \\
a_{12}=-a_{20} a_{11}, &
\end{array}
$$

and the reciprocal of an integrating factor is for this case

$$
\begin{aligned}
V(x, y)= & \left(1+\left(a_{20}+b_{11}\right) y\right)\left(1-a_{20} y\right)\left(\left(1-a_{20} y\right)^{2}\right. \\
& \left.+a_{11}\left(1-a_{20} y\right) x+\left(a_{21}-a_{20}^{2}-a_{20} b_{11}\right) x^{2}\right) .
\end{aligned}
$$

The associated constant of motion is analytic at the origin.

\section{General case}

We also carried out of calculations of section 1 for the cases $n=4$, $n=5$ and $n=6$.

We proposed the following form for $V(x, y)$ :

$$
V(x, y)=V_{1}(x, y) a_{n-1,1}+V_{2}(x, y),
$$

where $V_{1}(x, y)$ and $V_{2}(x, y)$ are independent of $a_{n-1,1}$. The regular form taken by these results has allowed us to propose a general compact expression for the integrability conditions and the reciprocal of an integrating factor, for arbitrary values of $n$.

For $2 \leq n \leq 6$, we found that the difference between the number of coefficients of the system (dimension): $(n-1)(n+4)$ and the number of integrability conditions $u_{n}$ has an arithmetic sequence progress:

$(n-1)(n+4)-u_{n}=3+2(n-2)$. Then we have $u_{n}=n^{2}+n-3$.

The general expressions for integrability conditions, $V(x, y)$ and $u_{n}$ that we induced from the results obtained for $n \leq 6$, is verified for $n=7$ and $n=8$.

So, we conjecture the following theorem: 
Theorem 2.1. Consider the system $(S)$, with $n$ arbitrary, and assums that $b_{n-1,0} \neq 0$. Under conditions

$$
\begin{aligned}
& a_{i, j}=(-1)^{j-1}\left(\frac{(n-i) !}{j !(n-(i+j)) !}(j-1) a_{20}^{2} b_{i-1,0}\right. \\
& \left.\quad+\frac{(n-i-1) !}{(j-1) !(n-(i+j)) !} a_{i, 1}\right) a_{20}^{j-1}, j \neq 0, \\
& a_{i, 0}=a_{20} b_{i-1,0}, \\
& a_{i, j}=(-1)^{j-1}\left(-\frac{(n-i-1) !}{j !(n-(i+j)) !} a_{20}\right. \\
& \left.\quad+\frac{(n-i-1) !}{(j-1) !(n-(i+j+1)) !} \frac{b_{n-1,1}}{b_{n-1,0}}\right) b_{i, 0} a_{20}^{j-1}, i+j<n, \quad j \neq 0, \\
& b_{i, j}=(-1)^{j-1} a_{20}^{j-1} b_{i, 0} \frac{b_{n-1,1}}{b_{n-1,0}}, \quad i+j=n, \quad j \neq 0, \\
& b_{n, 0}=0,
\end{aligned}
$$

$O(0,0)$ is a center and the reciprocal of an integrating factor is given by

$$
\begin{aligned}
V(x, y)= & \left(b_{n-1,0}+b_{n-1,1} y\right) \sum_{k=0}^{n}\left((n-k-1) a_{20}^{2} b_{n-1,0} b_{k-1,0}\right. \\
& \left.+a_{k, 1} b_{n-1,0}-a_{20} b_{k-1,0} b_{n-1,1}\right)\left(1-a_{20} y\right)^{n-k} x^{k}
\end{aligned}
$$

with $b_{i, j}=a_{i, j}=0$ if $i<0, j<0$ or $i+j>n$.

\section{Particular case.}

Suppose that $b_{n-1,0}=0$. Under conditions

$$
\begin{aligned}
a_{i, j}= & (-1)^{j-1}\left(\frac{(n-i) !}{j !(n-(i+j)) !}(j-1) a_{20}^{2} b_{i-1,0}\right. \\
& \left.+\frac{(n-i-1) !}{(j-1) !(n-(i+j)) !} a_{i, 1}\right) a_{20}^{j-1}, j \neq 0, \\
a_{i, 0}= & a_{20} b_{i-1,0}, \\
b_{i, j}= & (-1)^{j-1}\left(\left(\frac{(n-i) !}{j !(n-(i+j)) !}(j-1)\right.\right. \\
& \left.+\frac{(n-i-1) !}{(j-1) !(n-(i+j)) !}(i-1)\right) a_{20} \\
& \left.+\frac{(n-i-1) !}{(j-1) !(n-(i+j)) !} b_{11}\right) b_{i, 0} a_{20}^{j-1}, j \neq 0, \\
b_{n-1,1}= & a_{n, 0}=0,
\end{aligned}
$$


the origin is a center and the function $V(x, y)$ is given by

$$
V(x, y)=\left(1+\left((n-2) a_{20}+b_{11}\right) y\right) \sum_{k=0}^{n-1} \Omega_{k}(y) x^{k}
$$

where

$$
\Omega_{k}(y)=\left(-(k-1) a_{20} b_{k-1,0}-a_{20} b_{11} b_{k-1,0}+a_{k, 1}\right)\left(1-a_{20} y\right)^{n-k} .
$$

\section{Phase portrait of the system $\left(S_{1}\right)$}

Our purpose in this section is to demonstrate the following theorem

Theorem 3.1. If the conditions

$$
\begin{aligned}
& a_{20}>0, \quad b_{20}>0, \quad b_{21}>0, \\
& \Delta<0, \\
& r_{1}=a_{21}-a_{11} b_{20}+a_{20}^{2}+b_{20}^{2} \neq 0,
\end{aligned}
$$

and the conditions (25) are satisfied, the phase portrait of $\left(S_{1}\right)$ is homeomorphic to one of the configurations shown in Figures $1, \ldots, 4$.

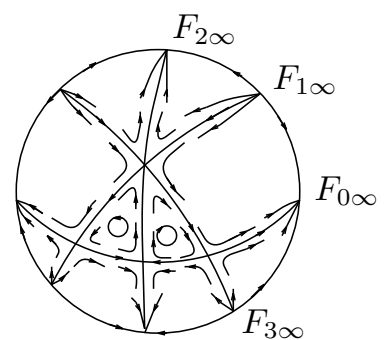

Figure 1

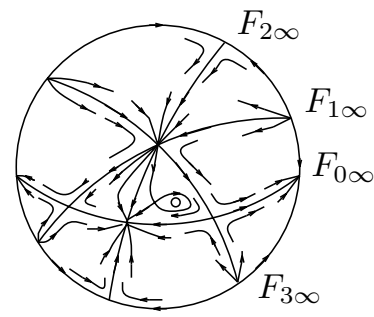

Figure 3

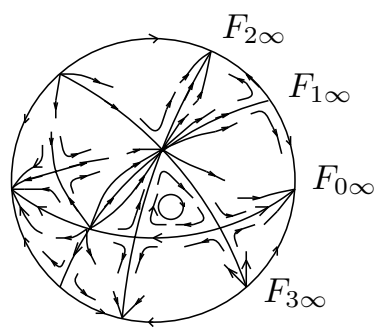

Figure 2

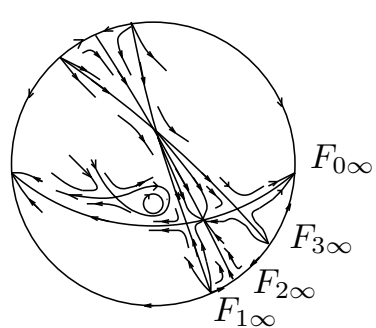

Figure 4

The phase portraits of the system $\left(S_{1}\right)$ associated with conditions (25) and (33). 
Figure 1 corresponds to the case $r_{1}<0$ and the others correspond to the case $r_{1}>0$. Here $\Delta$ is the discriminant of the homogeneous function $g_{2}(x, y)$ (see below).

Let us define

$$
\begin{aligned}
g_{1}(x, y)= & b_{20}+b_{21} y \\
g_{2}(x, y)= & -a_{20} b_{20}\left(a_{20} b_{20}+b_{21}\right) x^{3}+\left(a_{21} b_{20}-a_{20} b_{21}\right)\left(1-a_{20} y\right) x^{2} \\
& +a_{11} b_{20}\left(1-a_{20} y\right)^{2} x+b_{20}\left(1-a_{20} y\right)^{3} .
\end{aligned}
$$

When the conditions (25) are satisfied, we have

$$
V(x, y)=g_{1}(x, y) g_{2}(x, y) / b_{20}^{2} .
$$

About the functions $g_{1}(x, y)$ and $g_{2}(x, y)$, we have the following result.

Proposition 3.1. $g_{1}(x, y)=0$ and $g_{2}(x, y)=0$ are both algebraic particular integrals of the system $\left(S_{1}\right)$ when conditions (25) are satisfied.

Proof: By a straightforward calculation, we have

$$
\begin{aligned}
\frac{\partial g_{1}(x, y)}{\partial x} P(x, y)+\frac{\partial g_{1}(x, y)}{\partial y} Q(x, y)= & g_{1}(x, y)\left(-\frac{b_{21}}{b_{20}} x\left(1+b_{20} x-a_{20} y\right)\right) \\
\frac{\partial g_{2}(x, y)}{\partial x} P(x, y)+\frac{\partial g_{2}(x, y)}{\partial y} Q(x, y)= & g_{2}(x, y)\left(a_{11} b_{20}\left(1-a_{20} y\right) y\right. \\
& +\left(3 a_{20} b_{20}+\left(2 a_{21} b_{20}+a_{20} b_{21}\right) y\right) x \\
& \left.+3 a_{20} b_{20}^{2} x^{2}\right) / b_{20}
\end{aligned}
$$

These two equalities insure that $g_{1}(x, y)=0$ and $g_{2}(x, y)=0$ are algebraic particular integrals (or invariant curves).

Proposition 3.2. Let us suppose that $a_{20} b_{20}\left(a_{20} b_{20}+b_{21}\right) \neq 0$.

Let $\Delta$ be the discriminant of the polynomial $g_{2}(x, y)$. If $\Delta<0$ then the system $\left(S_{1}\right)$ has four invariant straight lines when conditions (25) are satisfied.

One of the straight lines is $L_{0}$, given by $b_{20}+b_{21} y=0$ and the others three have a common point of intersection.

Proof: We have already seen that $L_{0}$ is an invariant curve. If $\Delta<0$, we can write $g_{2}(x, y)$ as follows

$$
\begin{gathered}
g_{2}(x, y)=-a_{20} b_{20}\left(a_{20} b_{20}+b_{21}\right)\left(x-\alpha_{1}\left(1-a_{20} y\right)\right) \\
\left(x-\alpha_{2}\left(1-a_{20} y\right)\right)\left(x-\alpha_{3}\left(1-a_{20} y\right)\right),
\end{gathered}
$$


where $\alpha_{1}, \alpha_{2}$ and $\alpha_{3}$ are the zeros of the polynomial

$$
\begin{aligned}
h(z)= & z^{3}+\frac{a_{20} b_{21}-a_{21} b_{20}}{a_{20} b_{20}\left(a_{20} b_{20}+b_{21}\right)} z^{2}-\frac{a_{11} b_{20}}{a_{20} b_{20}\left(a_{20} b_{20}+b_{21}\right)} z \\
& -\frac{1}{a_{20}\left(a_{20} b_{20}+b_{21}\right)} .
\end{aligned}
$$

From equation (24) and after a direct calculation, we obtain the constant of motion

$$
\begin{array}{r}
I(x, y)=C_{1}(y)\left|x-\alpha_{1}\left(1-a_{20} y\right)\right|^{A_{1}}\left|x-\alpha_{2}\left(1-a_{20} y\right)\right|^{A_{2}} \\
\left|x-\alpha_{3}\left(1-a_{20} y\right)\right|^{A_{3}}=C,
\end{array}
$$

where

$$
\begin{aligned}
& A_{1}=-\frac{\alpha_{1}\left(1+\alpha_{1} b_{20}\right)}{\left(\alpha_{1}-\alpha_{2}\right)\left(\alpha_{3}-\alpha_{1}\right)}, \\
& A_{2}=-\frac{\alpha_{2}\left(1+\alpha_{2} b_{20}\right)}{\left(\alpha_{1}-\alpha_{2}\right)\left(\alpha_{2}-\alpha_{3}\right)}, \\
& A_{3}=-\frac{\alpha_{3}\left(1+\alpha_{3} b_{20}\right)}{\left(\alpha_{2}-\alpha_{3}\right)\left(\alpha_{3}-\alpha_{1}\right)},
\end{aligned}
$$

and $C_{1}(y)$ is a function of $y$ that is not necessary to give in an explicit form. This expression shows that the straight lines $L_{1}, L_{2}$ and $L_{3}$ defined by $x-\alpha_{i}\left(1-a_{20} y\right)=0(i=1,2,3)$, are particular integrals, obtained from the constant of motion for $C=0$ or $C=\infty$, depending on the sign of $A_{1}, A_{2}$ and $A_{3}$. These three invariant straight lines intersect at the point $M_{1}\left(0, \frac{1}{a_{20}}\right)$.

By using these results, we will study the phase portraits of $\left(S_{1}\right)$, with conditions (25) and (33).

Remark. The above first integral could actually be computed using computer algebra (MACSYMA).

Proposition 3.3. Under conditions (25) and (33) and with $r_{1}<0$, the only possible configuration of the critical points and the invariant straight lines is given by the Figure 5 while, under conditions (25) and (33) and with $r_{1}>0$, the possible configurations are given by Figures 6 , 7 and 8. 


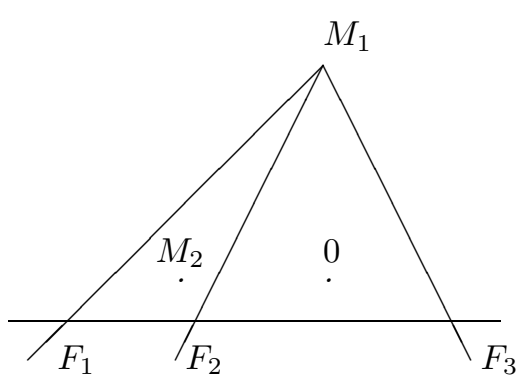

Figure 5

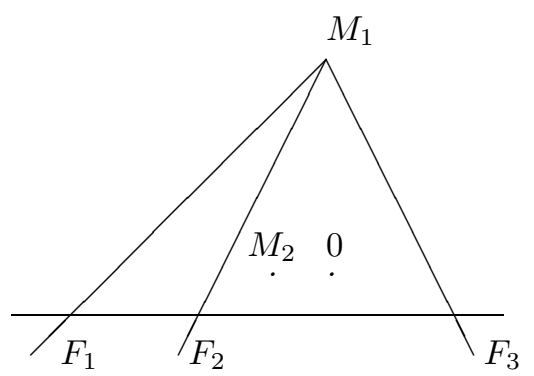

Figure 7

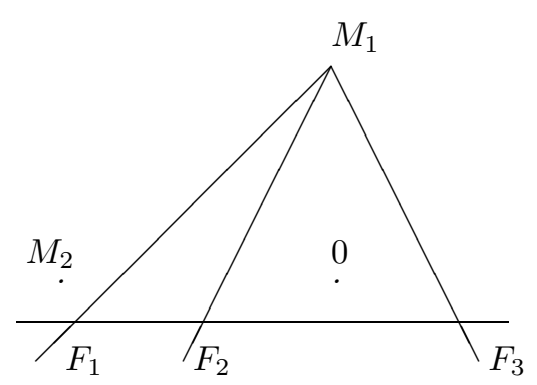

Figure 6

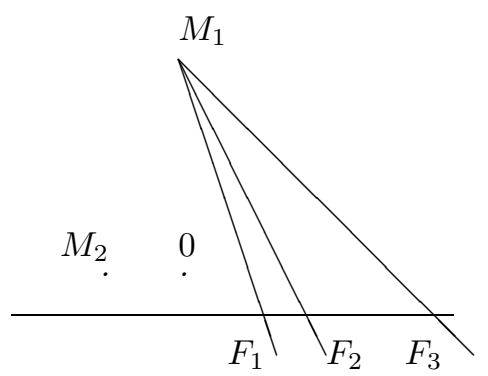

Figure 8

Proof: Under conditions (25), the polynomials $P(x, y)$ and $Q(x, y)$ of $\left(S_{1}\right)$ can be written as

$$
\begin{aligned}
P(x, y)= & y+a_{20} x^{2}+a_{11} x y-2 a_{20} y^{2}+a_{20} b_{20} x^{3} \\
& +a_{21} x^{2} y-a_{11} a_{20} x y^{2}+a_{20}^{2} y^{3} \\
Q(x, y)= & \frac{x\left(-1-b_{20} x+a_{20} y\right)\left(b_{20}+b_{21} y\right)}{b_{20}}
\end{aligned}
$$

From these expressions, we see that the system has six finite critical points

$$
\begin{array}{ccc}
O(0,0), & M_{1}\left(0, \frac{1}{a_{20}}\right), & M_{2}\left(-\frac{1}{b_{20}}, 0\right), \\
F_{1}\left(\beta_{1},-\frac{b_{20}}{b_{21}}\right), & F_{2}\left(\beta_{2},-\frac{b_{20}}{b_{21}}\right), & F_{3}\left(\beta_{3},-\frac{b_{20}}{b_{21}}\right),
\end{array}
$$

where $\beta_{1}, \beta_{2}$ and $\beta_{3}$ are the zeros of the polynomial

$$
\begin{aligned}
\left(-b_{21}^{3}\right) P\left(x,-\frac{b_{20}}{b_{21}}\right)= & b_{20}\left(a_{20} b_{20}+b_{21}\right)^{2}+a_{11} b_{20} b_{21}\left(a_{20} b_{20}+b_{21}\right) x \\
& +b_{21}^{2}\left(a_{21} b_{20}-a_{20} b_{21}\right) x^{2}-a_{20} b_{20} b_{21}^{3} x^{3} .
\end{aligned}
$$


The discriminant of this polynomial is $\Delta_{1}=b_{21}^{12} \Delta<0$.

By a direct calculation, we can verify that $F_{1}, F_{2}$ and $F_{3}$ are, respectively, the intersection points between $L_{1}, L_{2}$, and $L_{3}$ with $L_{0}$.

As $M_{2}$ is situated on the $x$ axis, the problem consists of determinating all the possible situations of $M_{2}\left(-\frac{1}{b_{20}}, 0\right)$ with respect to the intersection points of the straight lines $L_{1}, L_{2}$ and $L_{3}$ with the $x$ axe. The abscisses of this intersections are given by $\alpha_{1}, \alpha_{2}$ and $\alpha_{3}$, i.e the zeros of $g_{2}(x, 0)$ (and then also the zeros of $V(x, 0)$ ).

If we calculate $V(x, y)$ at the critical point $M_{2}$, we obtain

$$
V\left(-\frac{1}{b_{20}}, 0\right)=\frac{a_{21}-a_{11} b_{20}+a_{20}^{2}+b_{20}^{2}}{b_{20}^{2}}=\frac{r_{1}}{b_{20}^{2}} .
$$

Furthermore, the signs of $V(x, 0)$ are given by the Table 1

\begin{tabular}{|c|c|c|c|c|c|c|c|}
\hline$x$ & & $\alpha_{1}$ & & & & & $+\infty$ \\
\hline$V(x, 0)$ & + & & - & & & & - \\
\hline
\end{tabular}

Table 1

Using the fact that $V(0,0)=1>0$ we conclude that the origin $O$ is situated either in the intervalle $]-\infty, \alpha_{1}[$ or $] \alpha_{2}, \alpha_{3}[$.

1) Case where $O \in] \alpha_{2}, \alpha_{3}[$.

If $r_{1}>0$ then $V\left(-\frac{1}{b_{20}}, 0\right)>0$, and $M_{2}$ can be situated either in the intervalle $]-\infty, \alpha_{1}[$ or $] \alpha_{2}, 0[$.

If $r_{1}<0$ then $V\left(-\frac{1}{b_{20}}, 0\right)<0$ and $M_{2}\left(\frac{1}{b_{20}}, 0\right)$ must be in the intervalle $] \alpha_{1}, \alpha_{2}[$.

2) Case where $O \in]-\infty, \alpha_{1}[$.

We must consider only the case $r_{1}>0$, because if $r_{1}<0$ then $V\left(-\frac{1}{b_{20}}, 0\right)<0$ and $M_{2}$ must be situated in the intervalle $] \alpha_{1}, \alpha_{2}[$ or ]$\alpha_{3},+\infty\left[\right.$. This is a contradiction because $-\frac{1}{b_{20}}<0$.

If $r_{1}>0$ then $V\left(-\frac{1}{b_{20}}, 0\right)>0$, and $M_{2}$ must be in the intervalle ]$-\infty, 0[$.

Thus, we have the four possible configurations mentioned above. 


\section{Nature of critical points of $\left(S_{1}\right)$.}

As we said above, the system $\left(S_{1}\right)$ has six finite critical points when conditions (25) and (33) are satisfied. There are also four infinite critical points $F_{0 \infty}, F_{1 \infty}, F_{2 \infty}$ and $F_{3 \infty}$, located on the lines $L_{0}, L_{1}, L_{2}$ and $L_{3}$, respectively.

For the study of the nature of the critical points at infinity, we must apply the Poincaré's compactification of a plane vector field, defined by the central projection $[\mathbf{1 7}],[\mathbf{1 8}]: z=\frac{1}{x}, u=\frac{y}{x}(x \neq 0)$, which gives the transformed system $\left(S_{2}\right)$ :

$$
\begin{aligned}
\dot{z}=Q_{1}(u, z)= & -z\left(a_{20} b_{20}+a_{21} u+a_{20} z-a_{11} a_{20} u^{2}\right. \\
& \left.+a_{11} u z+a_{20}^{2} u^{3}-2 a_{20} u^{2} z+u z^{2}\right), \\
\dot{u}=P_{1}(u, z)= & -\left(a_{20} b_{20}+b_{21}\right) u-b_{20} z \\
& +\left(-a_{21}+\frac{a_{20} b_{21}}{b_{20}}\right) u^{2}-\frac{b_{21}}{b_{20}} u z-z^{2} \\
& +a_{11} a_{20} u^{3}-a_{11} u^{2} z-a_{20}^{2} u^{4}+2 a_{20} u^{3} z-u^{2} z^{2} .
\end{aligned}
$$

For the study of the nature of the critical points $F_{1 \infty}, F_{2 \infty}$ and $F_{3 \infty}$, it is convenient to introduce another transformation: $z=\frac{1}{y}, w=\frac{x}{y}$ that gives the system $S_{3}$ :

$$
\begin{aligned}
\dot{z}=Q_{2}(w, z)= & \frac{z w\left(-a_{20}+b_{20} w+z\right)\left(b_{21}+b_{20} z\right)}{b_{20}}, \\
\dot{w}=P_{2}(w, z)= & \left(a_{20}^{2} b_{20}-a_{11} a_{20} b_{20} w-2 a_{20} b_{20} z+\left(a_{21} b_{20}-a_{20} b_{21}\right) w^{2}\right. \\
& +a_{11} b_{20} w z+b_{20} z^{2}+b_{20}\left(a_{20} b_{20}+b_{21}\right) w^{3} \\
& \left.+b_{21} w^{2} z+b_{20}^{2} w^{3} z+b_{20} w^{2} z^{2}\right) / b_{20} .
\end{aligned}
$$

For obtaining $\left(S_{2}\right)$ and $\left(S_{3}\right)$, we rescaled the independent variable according to $t=z^{2} t^{\prime}$.

From $\left(S_{2}\right)$, we obtain $F_{0 \infty}(0,0)$ and $F_{i \infty}\left(\gamma_{i}, 0\right)(i=1,2,3)$, where $\gamma_{i}$ $(i=1,2,3)$ are the zeros of the polynomial

$$
\begin{aligned}
\left(-\frac{b_{20}}{u}\right) P_{1}(u, 0)= & b_{20}\left(a_{20} b_{20}+b_{21}\right)+\left(a_{21} b_{20}-a_{20} b_{21}\right) u \\
& -a_{11} a_{20} b_{20} u^{2}+a_{20}^{2} b_{20} u^{3}
\end{aligned}
$$

whose discriminant is: $\Delta_{2}=\frac{a_{20}^{4}}{\left(a_{20} b_{20}+b_{21}\right)^{2}} \Delta<0$.

Proposition 3.4. The points $F_{i}(i=1,2,3)$ and $F_{i \infty}(i=0,1,2,3)$ are saddle or node while $M_{2}$ is a saddle or a center. 
Proof: By a straightforward calculation, we can evaluate the eigenvalues associated to the critical points $F_{i}$ in terms of $\beta_{i}(i=1,2,3)$ as follows

$$
\begin{aligned}
\lambda_{1}\left(F_{i}\right)= & -\frac{\beta_{i}\left(a_{20} b_{20}+b_{21}+b_{20} b_{21} \beta_{i}\right)}{b_{20}} \\
\lambda_{2}\left(F_{i}\right)= & -\left(a_{11} b_{20}\left(a_{20} b_{20}+b_{21}\right)\right. \\
& \left.+2 b_{21}\left(a_{21} b_{20}-a_{20} b_{21}\right) \beta_{i}-3 a_{20} b_{20} b_{21}^{2} \beta_{i}^{2}\right) / b_{21}^{2} .
\end{aligned}
$$

For the critical points at infinity we find, from $\left(S_{2}\right)$

$$
\begin{aligned}
\lambda_{1}\left(F_{i \infty}\right)= & -a_{20} b_{20}-a_{21} \gamma_{i}+a_{11} a_{20} \gamma_{i}^{2}-a_{20}^{2} \gamma_{i}^{3}, \\
\lambda_{2}\left(F_{i \infty}\right)= & -a_{20} b_{20}-b_{21}-\left(2 a_{21}-\frac{2 a_{20} b_{21}}{b_{20}}\right) \gamma_{i} \\
& +3 a_{11} a_{20} \gamma_{i}^{2}-4 a_{20}^{2} \gamma_{i}^{3} \quad i=(1,2,3) .
\end{aligned}
$$

For $i=0$, we have: $\lambda_{1}\left(F_{0 \infty}\right)=-a_{20} b_{20}$ and $\lambda_{2}\left(F_{0 \infty}\right)=-\left(a_{20} b_{20}+b_{21}\right)$ which are both negative, thus $F_{0 \infty}$ is a stable node.

The above expressions for the eigenvalues associated to the points $F_{i \infty}(i=1,2,3)$ are rather complicated, so it is more easy to consider the system $\left(S_{3}\right)$. The critical points $F_{i \infty}$ of $\left(S_{2}\right)$ are transformed in the critical points $F_{i \infty}^{\prime}\left(\xi_{i}, 0\right)$ of $\left(S_{3}\right)$, where $\xi_{i}(i=1,2,3)$ are the zero of the polynomial

$$
\begin{aligned}
\left(b_{20}\right) P_{2}(w, 0)= & \left(a_{20}^{2} b_{20}-a_{11} a_{20} b_{20} w+\left(a_{21} b_{20}-a_{20} b_{21}\right) w^{2}\right. \\
& \left.+b_{20}\left(a_{20} b_{20}+b_{21}\right) w^{3}\right),
\end{aligned}
$$

whose discriminant is $\Delta_{3}=\Delta<0$. It is easy to show that the eigenvalues corresponding to the points $F_{i \infty}^{\prime}(i=1,2,3)$ are

$$
\begin{aligned}
& \lambda_{1}\left(F_{i \infty}^{\prime}\right)=\left(-a_{11} a_{20} b_{20}+2\left(a_{21} b_{20}-a_{20} b_{21}\right) \xi_{i}+3 b_{20}\left(a_{20} b_{20}+b_{21}\right) \xi_{i}^{2}\right) / b_{20}, \\
& \lambda_{2}\left(F_{i \infty}^{\prime}\right)=\frac{b_{21} \xi_{i}\left(-a_{20}+b_{20} \xi_{i}\right)}{b_{20}} .
\end{aligned}
$$

It is evident that $\lambda_{1}\left(F_{i}\right), \lambda_{2}\left(F_{i}\right), \lambda_{1}\left(F_{i \infty}\right)$ and $\lambda_{2}\left(F_{i \infty}\right)$ are real numbers.

Besides, the eigenvalues $\lambda_{1}\left(F_{i}\right)$ and $\lambda_{2}\left(F_{i}\right)$ are non-zero. It can be proved as follows.

Suppose that $\lambda_{1}\left(F_{i}\right)=0$, then $\beta_{i}=0$ or $\beta_{i}=-\frac{a_{20}}{b_{21}}-\frac{1}{b_{20}}$.

If $\beta_{i}=0$, then $F_{i}\left(0, \frac{b_{20}}{b_{21}}\right)$ is a critical point, but it is not possible, because the origin is a center and an invariant straight line cannot pass by such a point. 
If $\beta_{i}=-\frac{a_{20}}{b_{21}}-\frac{1}{b_{20}}$ then we have

$$
P\left(\beta_{i},-\frac{b_{20}}{b_{21}}\right)=-\frac{\left(a_{21}-a_{11} b_{20}+a_{20}^{2}+b_{20}^{2}\right)\left(a_{20} b_{20}+b_{21}\right)^{2}}{b_{20} b_{21}^{3}} \neq 0
$$

and the point $\left(\beta_{i},-\frac{b_{20}}{b_{21}}\right)$ cannot be a critical point. In consequence we have $\lambda_{1}\left(F_{i}\right) \neq 0(i=1,2,3)$.

If $\lambda_{2}\left(F_{i}\right)=0$, then the abscisse $\beta_{i}$ of the critical point $F_{i}$ must be a zero of $\lambda_{i}\left(F_{i}\right)$, then the remainder of the euclidian division of $P$ with $\lambda_{2}\left(F_{i}\right)$, calculated at the point $\left(\beta_{i},-\frac{b_{20}}{b_{21}}\right)$ must be a quantity independent from $\beta_{i}$. But a simple calculation shows that it is of the form $r_{2}=m_{1}+m_{2} \beta_{i}$, where

$$
m_{2}=-\frac{2 a_{20}}{9 b_{20}}-\frac{2 a_{21}^{2} b_{20}}{9 a_{20} b_{21}^{2}}-\frac{2 a_{11} a_{20} b_{20}^{2}}{3 b_{21}^{2}}+\frac{4 a_{21}}{9 b_{21}}-\frac{2 a_{11} b_{20}}{3 b_{21}} .
$$

In order to have a remainder independent of $\beta_{i}$, we must impose the condition $m_{2}=0$, but it is easy to show that this condition is incompatible with $\Delta<0$. In consequence, we have $\lambda_{2}\left(F_{i}\right) \neq 0(i=1,2,3)$.

Signs of $\lambda_{1}\left(F_{i}\right)$ and $\lambda_{2}\left(F_{i}\right)$.

Let us define the functions $k_{1}(x)$ and $k_{2}(x)$ as follows

$$
\begin{aligned}
k_{1}(x)= & -\frac{x\left(a_{20} b_{20}+b_{21}+b_{20} b_{21} x\right)}{b_{20}} \\
k_{2}(x)= & -\left(a_{11} b_{20}\left(a_{20} b_{20}+b_{21}\right)+2 b_{21}\left(a_{21} b_{20}-a_{20} b_{21}\right) x\right. \\
& \left.-3 a_{20} b_{20} b_{21}^{2} x^{2}\right) / b_{21}^{2} .
\end{aligned}
$$

It is evident that $k_{1}\left(\beta_{i}\right)=\lambda_{1}\left(F_{i}\right)$ and $k_{2}\left(\beta_{i}\right)=\lambda_{2}\left(F_{i}\right)$.

In the following, the identity $k_{2}(x)=\frac{\partial P}{\partial x}\left(x,-\frac{b_{20}}{b_{21}}\right)$ will be very useful for us. Let $x_{1}$ and $x_{2}$ be the zeros of $\frac{\partial P}{\partial x}\left(x,-\frac{b_{20}}{b_{21}}\right)$. We must consider two different cases.

\section{1) Case where $r_{1}<0$.}

For this case, we give the distribution of signs of the quantities that are relevant for the determination of the signs of $\lambda_{1}\left(F_{i}\right)$ and $\lambda_{2}\left(F_{i}\right)$ in 
Table 2.

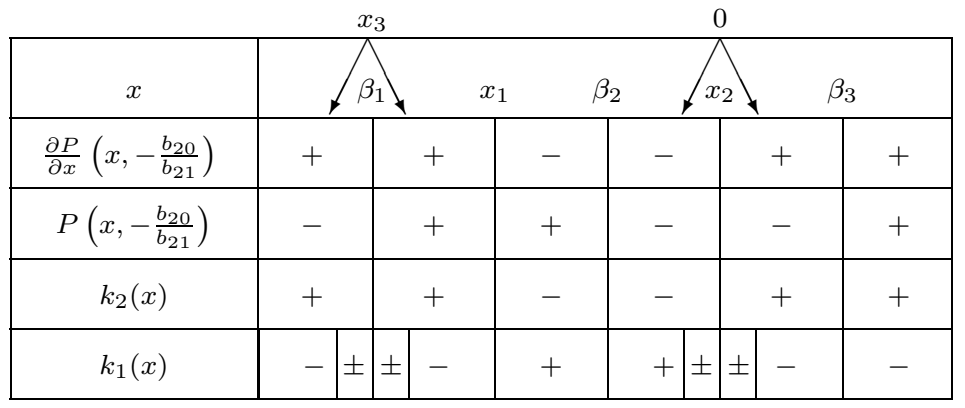

Table 2

For the signs of $k_{1}(x)$, we have already seen that $\left.O \in\right] \alpha_{2}, \alpha_{3}$ [ so $O \in$ ]$\beta_{2}, \beta_{3}[$. To be precise, $O \in] \beta_{2}, x_{2}\left[\right.$ if $a_{11} \geq 0$ and $\left.O \in\right] x_{2}, \beta_{3}$ [ if $a_{11}<0$. Obviously, the position of $O$ in the intervalle $] \beta_{2}, \beta_{3}$ [ is not important for the determination of the signs of $k_{1}(x)$ and $k_{2}(x)$ at $\beta_{i}(i=1,2,3)$, as can be seen from Table 2 .

The other zero of $k_{1}(x)$ is $x_{3}=-\frac{a_{20}}{b_{21}}-\frac{1}{b_{20}}<-\frac{1}{b_{20}}$.

As $\left.-\frac{1}{b_{20}} \in\right] \alpha_{1}, \alpha_{2}\left[\right.$, then $\left.-\frac{1}{b_{20}} \in\right] \beta_{1}, \beta_{3}[$.

Furthermore $P\left(x_{3},-\frac{b_{20}}{b_{21}}\right)=-\frac{r_{1}\left(a_{20} b_{20}+b_{21}\right)^{2}}{b_{20} b_{21}^{3}}>0$, which implies that $\left.x_{3} \in\right] \beta_{1}, \beta_{2}[$.

From these results, Table 2 follows.

We see that for the three points $F_{i}$, the signs of the two eigenvalues associated to each point are distinct. Then we conclude that $F_{1}, F_{2}$ and $F_{3}$ are saddle points.

2) Case where $r_{1}>0$.

We have $P\left(0,-\frac{b_{20}}{b_{21}}\right)=-\frac{b_{20}\left(a_{20} b_{20}+b_{21}\right)^{2}}{b_{21}^{3}}<0$ and $P\left(x_{3},-\frac{b_{20}}{b_{21}}\right)<0$. So we must consider three possibilities:

a) If $x_{3}<\beta_{1}<0$. From Table 3 , we conclude that $F_{1}$ is an unstable node, while $F_{2}$ and $F_{3}$ are saddle points.

Let us remark that $-\frac{1}{b_{20}}$ cannot be contained in the intervalle $] \beta_{2}, 0[$. 
Indeed we have that $\left.-\frac{1}{b_{20}} \in\right] x_{3}, \beta_{1}[$.

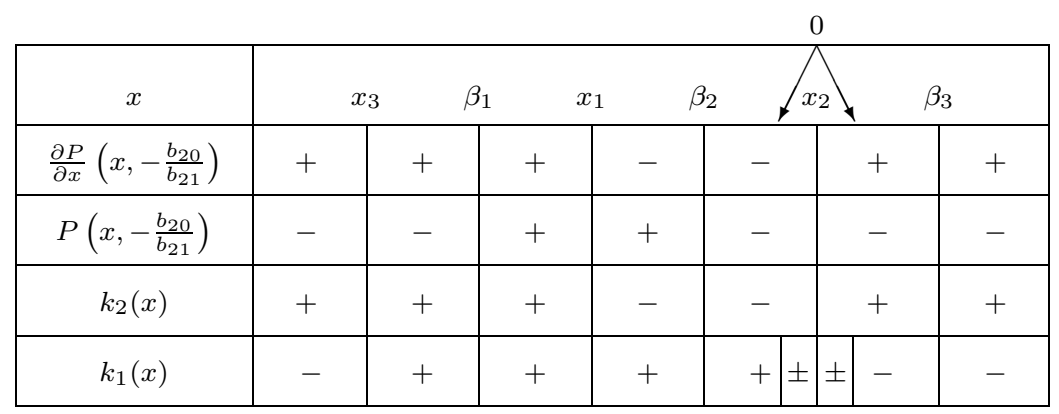

Table 3

b) If $\beta_{2}<x_{3}<0$. For this case we have Table 4, from which we conclude that $F_{2}$ is a stable node while $F_{1}$ and $F_{3}$ are saddle points.

\begin{tabular}{|c|c|c|c|c|c|c|c|c|}
\hline$x$ & \multicolumn{2}{|c}{$\beta_{1}$} & \multicolumn{7}{|c|}{0} \\
\hline$\frac{\partial P}{\partial x}\left(x,-\frac{b_{20}}{b_{21}}\right)$ & + & + & - & - & - & + & + \\
\hline$P\left(x,-\frac{b_{20}}{b_{21}}\right)$ & - & + & + & - & - & - & + \\
\hline$k_{2}(x)$ & + & + & - & - & - & + & + \\
\hline$k_{1}(x)$ & - & - & - & - & + & \multicolumn{2}{|c|}{$x_{2}$} & \multicolumn{2}{c|}{$\beta_{3}$} \\
\hline
\end{tabular}

Table 4

c) If $0<\beta_{1}<\beta_{2}<\beta_{3}$. For this case, the table of signs is given in Table 5 . We see that $F_{2}$ is a stable node, while $F_{1}$ and $F_{3}$ are saddle points.

\begin{tabular}{|c|c|c|c|c|c|c|c|c|}
\hline$x$ & \multicolumn{2}{|c}{$x_{3}$} & \multicolumn{2}{|c}{$\beta_{1}$} & $x_{1}$ & \multicolumn{2}{|c|}{$\beta_{2}$} & $x_{2}$ \\
\hline & \multicolumn{2}{|c|}{$\beta_{3}$} \\
\hline$\frac{\partial P}{\partial x}\left(x,-\frac{b_{20}}{b_{21}}\right)$ & + & + & + & + & - & - & + & + \\
\hline$P\left(x,-\frac{b_{20}}{b_{21}}\right)$ & - & - & - & + & + & - & - & + \\
\hline$k_{2}(x)$ & + & + & + & + & - & - & + & + \\
\hline$k_{1}(x)$ & - & + & - & - & - & - & - & - \\
\hline
\end{tabular}

Table 5 
For the study of the critical points at infinity, we apply the same procedure that for the points $F_{1}, F_{2}$ and $F_{3}$, but we treat with the transformed system $\left(S_{3}\right)$.

Is can be easily verified that $\lambda_{1}\left(F_{i \infty}^{\prime}\right)$ and $\lambda_{2}\left(F_{i \infty}^{\prime}\right)$ are both non-zero.

In order to study their signs, it is convenient to introduce the polynomials:

$k_{1}^{\prime}(w)=\left(-a_{11} a_{20} b_{20}+2\left(a_{21} b_{20}-a_{20} b_{21}\right) w+3 b_{20}\left(a_{20} b_{20}+b_{21}\right) w^{2}\right) / b_{20}$,

$k_{2}^{\prime}(w)=\frac{b_{21} w\left(-a_{20}+b_{20} w\right)}{b_{20}}$.

It is obvious that $k_{1}^{\prime}\left(\xi_{i}\right)=\lambda_{1}\left(F_{i \infty}^{\prime}\right)$ and $k_{2}^{\prime}\left(\xi_{i}\right)=\lambda_{2}\left(F_{i \infty}^{\prime}\right)$.

Furthermore, a direct calculation shows that

$k_{1}^{\prime}(w)=\frac{\partial P_{2}}{\partial w}(w, 0), \quad P_{2}(0,0)=a_{20}^{2}>0 \quad$ and $\quad P_{2}\left(\frac{a_{20}}{b_{20}}, 0\right)=\frac{a_{20}^{2} r_{1}}{b_{20}^{2}}$

We use the same method as above. We must consider two cases: $r_{1}<0$ and $r_{1}>0$ and obtain the following conclusion.

1) Case where $r_{1}<0$.

$F_{i \infty}^{\prime}(i=1,2,3)$ are unstable, stable and unstable nodes, respectively.

2) Case where $r_{1}>0$.

For this case we must consider three possibilities:

a) If $x_{4}>\gamma_{1}, F_{2 \infty}^{\prime}$ and $F_{3 \infty}^{\prime}$ are stable and unstable nodes, while $F_{1 \infty}^{\prime}$ is a saddle point.

b) If $0<x_{4}<\gamma_{2}, F_{1 \infty}^{\prime}, F_{3 \infty}^{\prime}$ are both unstable nodes while $F_{2 \infty}^{\prime}$ is a saddle.

c) If $0>\gamma_{1}>\gamma_{2}>\gamma_{3}, F_{1 \infty}^{\prime}, F_{3 \infty}^{\prime}$ are both unstable nodes and $F_{2 \infty}^{\prime}$ is a saddle.

The results that have been obtained above for the points $F_{i \infty}^{\prime}$ are also valid for the points $F_{i \infty}(i=1,2,3)$.

We can note that the Tables $6,7,8$ and 9 correspond respectively to Tables 2, 3, 4 and 5, respectively.

A direct calculation gives the eigenvalues associated to the critical point $M_{2}: \lambda_{1}\left(M_{2}\right)=\frac{\sqrt{r_{1}}}{b_{20}}$ and $\lambda_{2}\left(M_{2}\right)=\frac{\sqrt{r_{1}}}{b_{20}}$.

Therefore, if $r_{1}>0$ then $M_{2}$ is a saddle and if $r_{1}<0$, then $M_{2}$ is a center or a focus. 
Proposition 3.5. If $r_{1}<0$ then $M_{2}$ is a center.

Proof: If in the system $\left(S_{1}\right)$, we translate the origin at the point $M_{2}$ we obtain the system $\left(S_{4}\right)$

$$
\begin{aligned}
\dot{x}=P_{3}(x, y)= & \left(a_{20} b_{20} x+\left(a_{21}-a_{11} b_{20}+b_{20}^{2}\right) y-2 a_{20} b_{20}^{2} x^{2}\right. \\
& +b_{20}\left(a_{11} b_{20}-2 a_{21}\right) x y+a_{20} b_{20}\left(a_{11}-2 b_{20}\right) y^{2} \\
& \left.+a_{20} b_{20}^{3} x^{3}+a_{21} b_{20}^{2} x^{2} y-a_{11} a_{20} b_{20}^{2} x y^{2}+a_{20}^{2} b_{20}^{2} y^{3}\right) / b_{20}^{2}, \\
(36) \quad & -\frac{\left(-1+b_{20} x\right)\left(b_{20} x-a_{20} y\right)\left(b_{20}+b_{21} y\right)}{b_{20}^{2}} .
\end{aligned}
$$

As can be easily verified, the system $\left(S_{4}\right)$ admits the reciprocal of an integrating factor $\frac{1}{V_{3}}$, where $V_{3}(x, y)$ is given by

$$
\begin{aligned}
V_{3}(x, y)= & \left(b_{20}+b_{21} y\right)\left(-b_{20}\left(a_{21}-a_{11} b_{20}+a_{20}^{2}+b_{20}^{2}\right)\right. \\
& +b_{20}\left(3 a_{20}^{2} b_{20}+2 a_{21} b_{20}-a_{11} b_{20}^{2}+a_{20} b_{21}\right) x \\
& +a_{20}\left(a_{21} b_{20}-2 a_{11} b_{20}^{2}+3 b_{20}^{3}-a_{20} b_{21}\right) y \\
& -b_{20}^{2}\left(3 a_{20}^{2} b_{20}+a_{21} b_{20}+2 a_{20} b_{21}\right) x^{2} \\
& +2 a_{20} b_{20}\left(-a_{21} b_{20}+a_{11} b_{20}^{2}+a_{20} b_{21}\right) x y \\
& +a_{20}^{2} b_{20}^{2}\left(a_{11}-3 b_{20}\right) y^{2}+a_{20} b_{20}^{3}\left(a_{20} b_{20}+b_{21}\right) x^{3} \\
& \left.+a_{20} b_{20}^{2}\left(a_{21} b_{20}-a_{20} b_{21}\right) x^{2} y-a_{11} a_{20}^{2} b_{20}^{3} x y^{2}+a_{20}^{3} b_{20}^{3} y^{3}\right) .
\end{aligned}
$$

Furthermore, the associated constant of motion $I(x, y)$ defined by (29) is analytic in the neighbourhood of the origin $M_{2}: I(x, y) \sim \frac{b_{20}^{3}}{2 r_{1}}\left(b_{20} x^{2}-\right.$ $\left.2 a_{20} x y+\left(-a_{21}+a_{11} b_{20}-b_{20}^{2}\right) y^{2}\right)$.

Then, the level curves around the origin are ellipses and thus $M_{2}$ is a center.

The linear part of $\left(S_{1}\right)$ associated to the critical point $M_{1}$ is identically zero. Technic of blow up can be applied in order to determine the nature of such critical point [19]. In our study, the global phase portrait enable us to determine the nature of this critical point, as can be seen from the figures, and then the Theorem 3 follows.

Much of calculations performed in this work have been made with Mathematica computer algebra system.

\section{References}

1. H. Giacomini and M. Ndiaye, Sufficient conditions for the existence of a center in polynomial systems of arbitrary degree, Publ. Mat. 40 (1996), 205-214. 
2. D. Schlomiuk, J. Guckenheimer and R. Rand, Integrability of plane quadratic vector fields, Expoxition Math. 8 (1990), 3-25.

3. D. Schlomiuk, Algebraic particular integrals, Integrability and the problem of the center, Trans. Amer. Math. Soc. 338 (1993), p. 799.

4. H. ŻOLADEK, Quadratic systems with center and their perturbations, J. Differential Equations 109 (1994), 223-273.

5. V. A. Lunkevich And S. K. Sibirski, On the condition for a center, Differential'nye Uravneniya 1 (1965), 53-66.

6. N. G. Lloyd And J. M. Pearson, Computing center conditions for certain cubic systems, J. Comput. Appl. Math. 40 (1992), 323-336.

7. H. ŻOLADEK, The classification of reversible cubic systems with center, Topol. Methods Nonlinear Anal. 4 (1994), 79-136.

8. J. Sokulsky, The beginning of the classification of Darboux integrals for cubic systems, Preprint, Warsaw (1993).

9. H. Giacomini and M. Viano, Determination of limit cycles for two-dimensional dynamical systems, Phys. Rev. E52 (1995), p. 222.

10. J. Chavarriga, Integrable systems in the plane with center type linear part, Appl. Math. (Warsaw) 22 (1994), 285-309.

11. C. J. Christopher And N. G. Lloyd, On the paper of Jin and Wang concerning the conditions for a center in certain cubic systems, Bull. London Math. Soc. 22 (1990), 5-12.

12. G. Darboux, Mémoire sur les équations difféntielles algébriques du premier ordre et du premier degré (Mélanges), Bull. Sci. Math. 2 (1878), 60-96, 123-144, 151-200.

13. M. J. Prelle And M. D. Singer, Elementary first integral of differential equations, Trans. Amer. Math. Soc. 279 (1983), 215-229.

14. D. Schlomiuk, Elementary first integrals of differential equations and invariant algebraic curves, Exposition. Math. 11 (1993), 433454.

15. D. SCHLOMIUK, "Algebraic and geometric aspects of the theory of polynomial vector fields," ed., Kluwer Academic Press, NATO ASI Series, Series C-408, 1993, pp. 429-467.

16. C. J. Christopher, Invariant algebraic curves and conditions for a center, Proc. Roy. Soc. Edinburgh Sect. A 124 (1994).

17. H. Poincaré, Mémoire sur les courbes définies par les équations différentielles, Journal de Math.(3) 37 (1881), 375-422; 8, (1882), 251-296; "Oeuvres de Henri Poincaré," vol. I, Gauthier-Villars, Paris, 1951, pp. 3-84. 
18. L. PERKo, "Differential equations and dynamical systems," Springer-Verlag, New York, 1991.

19. A. A. Andronov, E. A. Leontovich, I. I. Gordon And A. G. MAIER, "Qualitative theory of second-order dynamical systems," John Wiley \& Sons, New York, 1973.

\author{
Laboratoire de modèles de Physique Mathématique \\ Faculté des Sciences et Techniques \\ Université de Tours \\ 37200 Tours \\ FRANCE
}

Primera versió rebuda el 23 d'Octubre de 1995, darrera versió rebuda el 10 de Novembre de 1995 